\title{
FMRP sustains presynaptic function via control of activity-dependent bulk endocytosis
}

Katherine Bonnycastle ${ }^{1,2,3}$, Peter C. Kind ${ }^{1,2,3}$ and Michael A. Cousin ${ }^{1,2,3^{*}}$

1. Centre for Discovery Brain Sciences, Hugh Robson Building, George Square, University of Edinburgh, Edinburgh, Scotland, UK EH8 9XD.

2. Patrick Wild Centre, Hugh Robson Building, George Square, University of Edinburgh, Edinburgh, Scotland, UK EH8 9XD.

3. Simons Initiative for the Developing Brain, Hugh Robson Building, George Square, University of Edinburgh, Edinburgh, Scotland, UK EH8 9XD.

\section{* Correspondence -}

Centre for Discovery Brain Sciences, Hugh Robson Building, George Square, University of Edinburgh, Edinburgh, Scotland, UK EH8 9XD.

Email - M.Cousin@ed.ac.uk.

ORCID - 0000-0002-1762-160X 


\title{
SUMMARY
}

Fragile $X$ syndrome ( $F X S$ ) is caused by loss of fragile $X$ mental retardation protein (FMRP). Bonnycastle et al show that FMRP is specifically required for activity-dependent bulk endocytosis (ADBE), revealing 1) FMRP sustains neurotransmitter release and 2) intervention via ADBE may correct circuit hyperexcitabilty in FXS.

\begin{abstract}
Synaptic vesicle (SV) recycling defects are linked to neurodevelopmental disorders, including fragile $X$ syndrome (FXS), which results from loss of fragile $X$ mental retardation protein (FMRP) encoded by the FMR1 gene. Hyperexcitability of neuronal circuits is a key feature of FXS, therefore we investigated whether SV recycling was affected by the absence of FMRP during increased neuronal activity. We revealed that primary neuronal cultures from a Fmr1 knockout rat model display a specific defect in activity-dependent bulk endocytosis (ADBE). This defect resulted in an inability of Fmr1 knockout neurons to sustain SV recycling during high frequency stimulation. Using a molecular replacement strategy, we also revealed that a human FMRP mutant that cannot bind BK channels failed to correct ADBE dysfunction in knockout neurons, however this dysfunction was corrected by BK channel agonists. Therefore, FMRP performs a key role in sustaining neurotransmitter release via selective control of $A D B E$, suggesting intervention via this endocytosis mode may correct hyperexcitabiltiy observed in FXS.
\end{abstract}

\section{SHORT TITLE}

FMRP sustains neurotransmission via control of $A D B E$ 


\section{INTRODUCTION}

Information communication between neurons requires the activity-dependent, synchronous fusion of neurotransmitter-containing synaptic vesicles (SVs). SVs mobilised by action potentials (APs) are termed the recycling pool, which is subdivided into the readily releasable pool (RRP, comprising primed fusionready SVs) and the reserve pool (mobilised during high neuronal activity (Alabi and Tsien, 2012)). Several SV endocytosis modes are sequentially recruited by increasing stimulus intensity to replenish these SV pools (Chanaday et al., 2019). Ultrafast endocytosis is dominant during sparse neuronal activity (Watanabe et al., 2013), whereas clathrin-mediated endocytosis becomes prevalent during AP trains (Granseth et al., 2006). Both ultrafast and clathrin-mediated endocytosis saturate during heightened neuronal activity (Lopez-Murcia et al., 2014; Soykan et al., 2017), and under these conditions, activitydependent bulk endocytosis (ADBE) is the dominant endocytosis mode (Clayton et al., 2008). ADBE generates large endosomes directly from the plasma membrane, from which SVs are formed to replenish the reserve pool (Cheung et al., 2010; Richards et al., 2000).

The translation repressor fragile $X$ mental retardation protein (FMRP) is located in central nerve terminals, where it controls the expression of numerous presynaptic proteins (Darnell and Klann, 2013). However, it also has protein synthesis-independent presynaptic functions, such as mediating ion channel gating, activation, and density (reviewed in Ferron, 2016). For example, FMRP interacts with Slack potassium channels (Brown et al., 2010), N-type calcium channels (Ferron et al., 2014) and large conductance voltage and calcium-gated big potassium (BK) channels (Deng et al., 2013; Deng and Klyachko, 2016; Kshatri et al., 2020). The absence of FMRP at CA3-CA1 hippocampal synapses leads to reduced BK channel activity and excessive AP broadening (Deng et al., 2013; Wang et al., 2014; Deng and Klyachko, 2016), resulting in increased presynaptic calcium influx during high frequency stimulation (Deng et al., 2011; Deng et al., 2013). These synapses also display altered short-term plasticity, but only during periods of heightened activity (Deng et al., 2011; Klemmer et al., 2011). Therefore, presynaptic phenotypes that occur in the absence of FMRP are only revealed during intense neuronal activity (Deng et al., 2011; Deng et al., 2013; Ferron et al., 2014).

Hyperexcitability of neuronal circuits is a key feature of fragile $X$ syndrome (FXS) (Booker et al., 2019; Das Sharma et al., 2020), one of the most common monogenic causes of intellectual disability (ID) and autism spectrum disorder (Mefford et al., 2012). Most FXS cases are caused by a CGG trinucleotide expansion in the 5' untranslated region of the FMR1 gene which encodes FMRP, leading to hypermethylation of the promoter and silencing of the FMR1 gene. A point mutation which disrupts polyribosome binding (I304N), is sufficient to cause FXS (Feng et al., 1997), whereas a different mutation results in mutant FMRP that is unable to bind to and regulate BK channels (R138Q).

Since FMRP may be required for accurate presynaptic function, specifically during intense neuronal activity, we determined whether SV recycling was disproportionately impacted under these conditions in primary neuronal cultures derived from a Fmr1 knockout (KO) rat model (Asiminas et al., 2019). No significant defect in either SV exocytosis or endocytosis was observed, however Fmr1 KO neurons displayed a robust defect in ADBE. This defect reduced presynaptic performance during periods of intense neuronal activity. Finally, molecular replacement studies with FMRP mutants revealed that the $A D B E$ defect was due to loss of BK channel interactions and that BK channel activators could restore normal function in Fmr1 KO neurons. 


\section{RESULTS AND DISCUSSION}

\section{Fmr1 KO neurons display no obvious defect in SV recycling}

FMRP is proposed to control SV fusion (Ferron et al., 2014), presynaptic AP duration (Deng et al., 2011), and short-term synaptic plasticity (Deng et al., 2011; Klemmer et al., 2011) in different murine models. To determine whether SV exocytosis or endocytosis were altered in a newly generated Fmr1 KO rat model (Asiminas et al., 2019), we examined SV recycling using synaptophysin-pHluorin (sypHy) in primary hippocampal cultures from either KO or wild-type (WT) littermate controls. SypHy consists of the SV protein synaptophysin with a pH-sensitive EGFP (pHluorin) fused to an intraluminal loop (Granseth et al., 2006). Since sypHy reports the $\mathrm{pH}$ of its immediate environment, an exocytosis-dependent increase in its fluorescence signal is observed during SV fusion (Fig. 1A, B). After endocytosis, sypHy fluorescence is quenched by SV acidification. This loss of fluorescence is an estimate of the kinetics of SV endocytosis, since this is rate-limiting (Atluri and Ryan, 2006; Granseth et al., 2006). When WT neurons were challenged with a 300 AP train delivered at $10 \mathrm{~Hz}$, they displayed a characteristic sypHy response, with an evoked fluorescence increase followed by an exponential post-stimulation decrease to baseline. To determine the amount of exocytosis as a proportion of the total SV pool, fluorescence traces were normalised to the sypHy response in the presence of $\mathrm{NH}_{4} \mathrm{Cl}$ (to reveal the maximal unquenched signal; Fig. 1B). No significant difference was observed when WT and KO were compared (Fig. 1C). The lack of effect was confirmed using the vacuolar-type ATPase inhibitor bafilomycin A1 to isolate SV exocytosis without the confound of SV endocytosis (Sankaranarayanan and Ryan, 2001) (Fig. S1A, B). The time constant, T, of SV endocytosis was also not significantly different between WT and KO neurons (Fig. 1D). Therefore, loss of FMRP does not impair SV exocytosis or endocytosis during low frequency stimulation.

Previous studies examining loss of FMRP function revealed presynaptic defects during high frequency stimulation (Deng et al., 2011; Wang et al., 2014; Ferron et al., 2014). Therefore, we next examined sypHy responses evoked by a train of $400 \mathrm{APs}$ delivered at $40 \mathrm{~Hz}$ (Fig. 1E-G). However, there was again no difference in either the extent of SV exocytosis (Fig. 1F, Fig. S1C, D) or the kinetics of SV endocytosis (Fig. 1G). Taken together, this indicates that $F m r 1$ deletion does not play a significant role in SV recycling during either low or high frequency activity.

An increase in SV pools may partially explain the observed lack of effect on SV exocytosis in Fmr1 KO cultures. To determine this, we used bafilomycin A1 in sypHy-transfected neurons to isolate SV fusion (Fig. $1 \mathrm{H}-\mathrm{K})$. In these experiments, the RRP was mobilised by $40 \mathrm{APs}(20 \mathrm{~Hz})$, and the remainder of the recycling pool by 1600 APs $(20 \mathrm{~Hz}$, Fig. $1 \mathrm{H})$. The resting pool (which cannot be mobilised by APs) was revealed by application of $\mathrm{NH}_{4} \mathrm{Cl}(\mathrm{Fig} .1 \mathrm{H})$. We observed no difference either in the size of the total SV pool (Fig. 1I) or RRP between genotypes (Fig. 1J), however the SV recycling pool was increased in KO cultures (Fig. 1K). Thus, the proportions of SVs in different functional pools are shifted in KO cultures, with more SVs in the recycling pool at the expense of those in the resting pool. This adaptation suggests that $F m r 1 \mathrm{KO}$ nerve terminals compensate for reduced functionality by redistributing SVs to pools that are accessible to neuronal activity.

\section{Fmr1 KO neurons have defective ADBE}

The increased SV recycling pool in Fmr1 KO neurons could reflect a compensation for a presynaptic defect. Since presynaptic deficiencies in the absence of FMRP are only revealed during high frequency stimulation (Deng et al., 2011; Wang et al., 2014; Ferron et al., 2014), we next assessed whether a process that is dominant under these conditions, ADBE, was affected in Fmr1 KO neurons. ADBE was monitored optically using uptake of $40 \mathrm{kDa}$ tetramethylrhodamine (TMR)-dextran, a fluid phase marker that is selectively accumulated via this endocytosis mode (Clayton et al., 2008). WT and Fmr1 KO neurons were challenged with a train of $400 \mathrm{APs}(40 \mathrm{~Hz})$ to maximally trigger ADBE in the presence of TMR-dextran (Clayton et al., 2008; Nicholson-Fish et al., 2015). The number of nerve terminals that 
perform ADBE were revealed as discrete TMR-dextran fluorescent puncta (Fig. 2A, B). We observed a significant decrease in TMR-dextran puncta in Fmr1 KO neurons relative to WT littermate controls (Fig. 2C). Importantly, this was not due to synapse loss in the Fmr1 KO cultures, since there was no difference in nerve terminal numbers (measured by staining for the SV protein SV2A, Fig. S2). Taken together, these results reveal a small but robust decrease in the number of nerve terminals undergoing ADBE, suggesting that FMRP may be required for this mode of endocytosis.

TMR-dextran uptake does not report the extent to which ADBE occurs in each nerve terminal (Clayton et al., 2008). Therefore, to confirm the ADBE defect in Fmr1 KO neurons, we applied the fluid phase marker horse radish peroxidase (HRP) during AP stimulation ( $40 \mathrm{~Hz} 10 \mathrm{~s}$, Fig. 2D, E). Quantification of the number of HRP-labelled endosomes revealed a significant reduction in Fmr1 KO neurons compared to WT (Fig. 2F), whereas the endosome area was not significantly different (Fig. S3). This reduction in bulk endosome generation reveals that Fmr1 KO neurons display a specific defect in both the extent and prevalence of ADBE during intense neuronal activity.

\section{Fmr1 KO neurons display decreased presynaptic performance}

Since ADBE is the dominant endocytosis mode during intense neuronal activity (Clayton et al., 2008), the impact of its dysfunction on SV exocytosis may only be revealed during patterns of high frequency stimulation. To determine this, we monitored the amount of sypHy that visited the plasma membrane as a surrogate of neurotransmitter release, in response to multiple high frequency AP trains (Fig. 3A, B). We predicted that $F m r 1 \mathrm{KO}$ neurons would be unable to sustain performance to the same extent as WT, due to fewer new SVs being provided via ADBE (Nicholson-Fish et al., 2015). Cultures were stimulated with four high frequency AP trains $(40 \mathrm{~Hz} 10 \mathrm{~s})$ separated by 5 min intervals. WT neurons displayed a sequential decrease in the extent of the evoked sypHy response, consistent with a depletion of SVs available for exocytosis. When the same protocol was performed for Fmr1 KO neurons, the sypHy response during the final stimulation was significantly lower when compared to WT (Fig. 3B). This suggests FMRP sustains neurotransmitter release during increased neuronal activity via its control of ADBE.

\section{BK channel interactions are required for FMRP function in ADBE}

To determine the mechanism underlying the control of ADBE by FMRP, we determined whether specific loss of function mutants could rescue TMR-dextran uptake in Fmr1 KO cultures. First, cultures from Fmr1 $\mathrm{KO}$ rats or WT littermate controls were transfected with either an empty fluorescent mCerulean vector (mCer, empty) or mCer plus EGFP-FMRP (FMRP). Overexpression of FMRPwT did not affect evoked TMR-dextran uptake in WT neurons (Fig. 4A, B), indicating that excess FMRP has no detrimental effect on ADBE. It also signified that additional FMRP does not enhance ADBE, as observed with constitutively active Rab11 mutants (Kokotos et al., 2018). Importantly, FMRPWT expression fully rescued the impairment in TMR-dextran uptake in Fmr1 KO neurons (Fig. 4B).

We exploited the ability of FMRPWT to rescue TMR-dextran uptake to perform molecular replacement

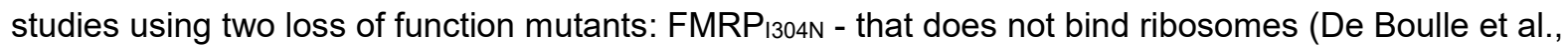
1993) and FMRP $P_{R 138 Q}$ - that does not bind BK channels (Myrick et al., 2015; Kshatri et al., 2020) (Fig. 4C). When these experiments were performed, FMRP ${ }_{W T}$ restored TMR-dextran uptake in Fmr1 KO cultures as previously observed (Fig. 4D). TMR-dextran uptake in FMRPI304N expressing neurons was not significantly different to neurons expressing FMRPWT (Fig. 4D), indicating that FMRP-dependent control of protein translation is dispensable for ADBE. However, expression of FMRPR138Q failed to restore TMRdextran uptake in Fmr1 KO neurons, with levels equivalent to those observed with an empty vector (Fig. 4D). These results strongly suggest that FMRP controls ADBE through interactions with BK channels. 
The inability of $F M R P_{R 138 Q}$ to rescue ADBE in Fmr1 KO neurons supports the hypothesis that presynaptic defects in the absence of FMRP are translation-independent (Deng and Klyachko, 2016). The R138Q mutation is found in individuals presenting with ID (and in some cases FXS phenotypes, seizures and autism) (Myrick et al., 2015; Sitzmann et al., 2018; Diaz et al., 2018; Collins et al., 2010). However, given its prevalence in the general population (identified in 20 subjects, of which 9 were male, that display no discernable ID - https://gnomad.broadinstitute.org) it is unlikely that this mutation directly contributes to FXS.

\section{BK channel activation corrects ADBE defects in Fmr1 KO neurons}

The absence of $A D B E$ rescue by $F M R P_{R 138 Q}$ suggests that $B K$ channel modulation by FMRP may be important for this endocytosis mode. Therefore, we investigated whether altering BK channel activity impacted ADBE. First, we determined whether enhancing channel activity with the activator NS 11021 could restore ADBE in Fmr1 KO neurons. BK channel activation with NS 11021 did not affect TMRdextran uptake in WT neurons when compared to a vehicle control (Fig. 4E). It did however restore TMRdextran uptake in Fmr1 KO neurons to WT levels (Fig. 4E). Therefore, activation of BK channels is sufficient to correct dysfunctional ADBE in Fmr1 KO neurons.

To determine whether loss of BK channel function was responsible for defective ADBE in Fmr1 KO neurons, we attempted to mimic the deficit using the BK channel antagonist Paxilline in WT hippocampal cultures. Interestingly, this manoeuvre did not impact activity-dependent TMR-dextran uptake (Fig. 4F). Taken together, these results suggest that BK channel dysfunction is not responsible for ADBE defects in Fmr1 KO neurons, however BK channel activation can correct this fault.

\section{ADBE acts as a rheostat to tune neuronal excitability}

It is likely that the observed reduction in ADBE reflects a compensatory or homeostatic mechanism to counteract the increased excitability in FXS and Fmr1 KO models (Booker et al., 2019; Das Sharma et al., 2020; Zhang et al., 2014). BK channel openers decrease hyperexcitability in Fmr1 KO models both in vitro and in vivo by increasing hyperpolarization, resulting in decreased calcium influx in nerve terminals.(Zhang et al., 2014; Hebert et al., 2014). The correction of ADBE by BK channel activation in this study, suggests that this is the most likely mechanism of action. Although it appears counterintuitive that an endocytosis mode which is triggered by high neuronal activity should be depressed by hyperexcitability, ADBE is reduced by stimulus frequencies above $40 \mathrm{~Hz}$ (Clayton et al., 2008). This inhibition may result from excess activity-dependent calcium influx, since this inhibits various forms of SV endocytosis in both large atypical and small classical central nerve terminals (Cousin and Robinson, 2000; Wu and Wu, 2014; von Gersdorff and Matthews, 1994; Leitz and Kavalali, 2016).

A homeostatic reduction in ADBE in response to increased hyperexcitability would not be a homogenous adaptation across the brain, since neurons that display high firing rates would be disproportionately impacted. Therefore, even if reduced ADBE is not a causal mechanism in FXS, it may still be a valuable therapeutic intervention point to correct hyperexcitability in specific circuits (Booker et al., 2019; Das Sharma et al., 2020). Future studies are now required to determine whether modulation of ADBE can sculpt circuit activity in FXS (and other autism models that display hyperexcitability - such as SynGAP haploinsufficiency disorder (Gamache et al., 2020)). However, irrespective of whether ADBE dysfunction directly contributes to FXS, the role of FMRP in the control of this key event provides valuable new information into the mechanisms of SV recycling that regulate presynaptic function. 

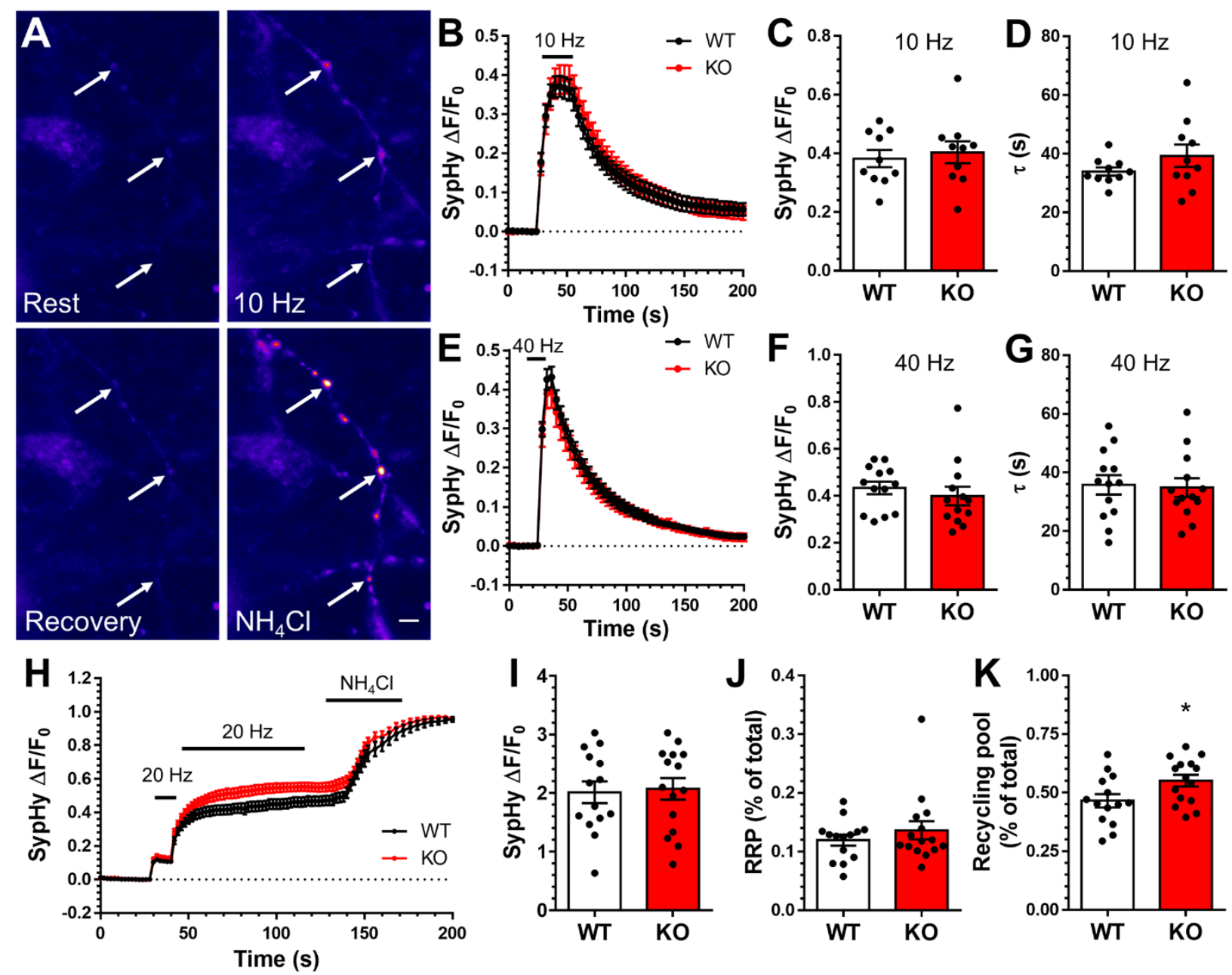

Figure 1. Fmr1 KO neurons display no defect in SV recycling.

Hippocampal neurons from either Fmr1 KO or WT littermate controls were transfected with sypHy on DIV 7 and imaged DIV 13-15. A-G) Neurons were challenged with a train of APs (either $10 \mathrm{~Hz} 30 \mathrm{~s}$, B-D; or 40 $\mathrm{Hz} 10 \mathrm{~s}, \mathrm{E}-\mathrm{G}$ ) before exposure to $\mathrm{NH}_{4} \mathrm{Cl} 3$ min later. A) Representative images of SypHy-transfected nerve terminals (indicated by arrows) during this experiment (Rest, $10 \mathrm{~Hz}$, Recovery and $\mathrm{NH}_{4} \mathrm{Cl}$, scale bar $=5 \mu \mathrm{m}$ ). B, E) Mean traces displaying the average sypHy response of WT (black) and KO (red) neurons in response to $10 \mathrm{~Hz}(B)$ or $40 \mathrm{~Hz}(E)$ stimulation $\left(\triangle F / F_{0}\right.$ as a fraction of the total SV pool, revealed by $\mathrm{NH}_{4} \mathrm{Cl}$ ). Bar indicates stimulation period. C, F) Mean sypHy peak heights during either $10 \mathrm{~Hz}(\mathrm{C})$ or $40 \mathrm{~Hz}$ (F) stimulation. D, G) Mean sypHy retrieval time constants (T) following either $10 \mathrm{~Hz}(D)$ or $40 \mathrm{~Hz}(\mathrm{G})$ stimulation. H-K) SypHy transfected neurons were challenged with two AP trains $(20 \mathrm{~Hz}, 2 \mathrm{~s}$ and $20 \mathrm{~Hz}$ $80 \mathrm{~s}$ ) before exposure to $\mathrm{NH}_{4} \mathrm{Cl}$ in the presence of $1 \mu \mathrm{M}$ bafilomycin $\mathrm{A} 1 . \mathrm{H}$ ) Mean traces display the average sypHy fluorescent response of WT (black) and $\mathrm{KO}(\mathrm{red})$ neurons $\left(\Delta \mathrm{F} / \mathrm{F}_{0}\right.$, normalised to the total $\mathrm{SV}$ pool, revealed by $\mathrm{NH}_{4} \mathrm{Cl}$ ). Bars indicate stimulation period. I) Mean sypHy fluorescence during exposure to $\mathrm{NH}_{4} \mathrm{Cl}$. J) Mean sypHy peak height during $20 \mathrm{~Hz} 2 \mathrm{~s}$ stimulation (RRP, \% of total pool). K) Mean sypHy peak height during $20 \mathrm{~Hz} 80 \mathrm{~s}$ stimulation (Recycling pool, \% of total pool. B-K) All data \pm SEM. For statistical information see Table S1. 

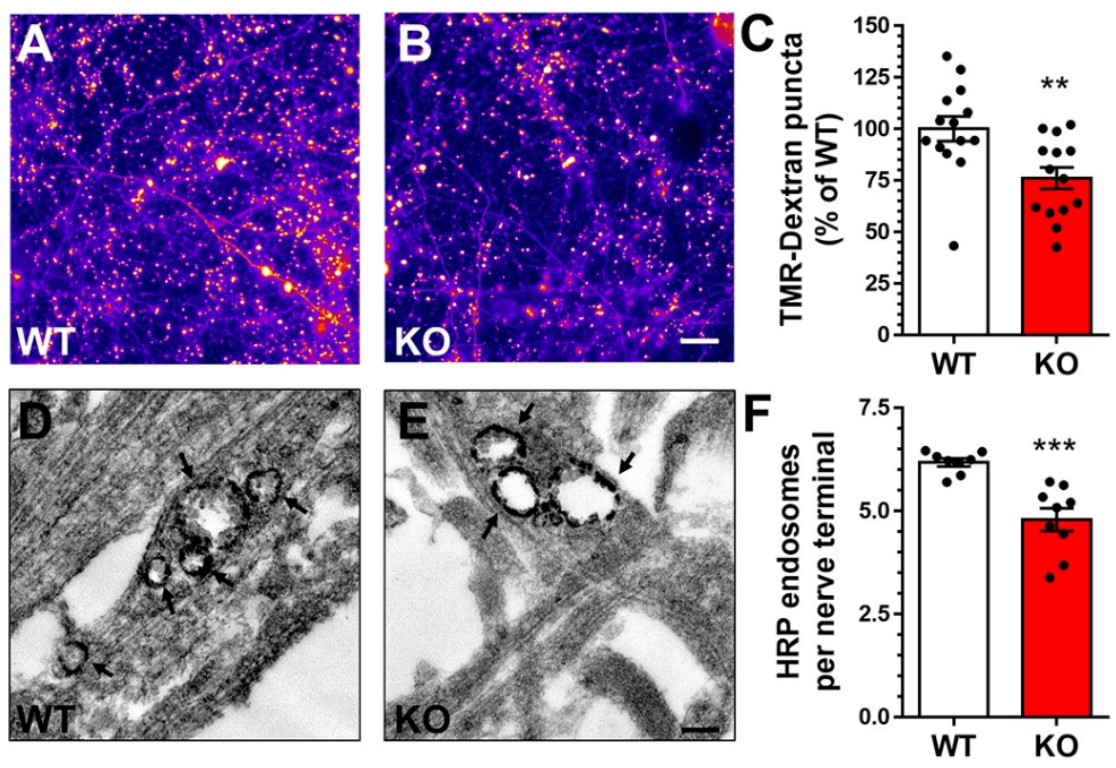

Figure 2. Fmr1 KO neurons have defective ADBE.

A-C) Hippocampal neurons from either Fmr1 KO or WT littermate controls were challenged with a $40 \mathrm{~Hz}$ $10 \mathrm{~s}$ stimulus in the presence of $50 \mu \mathrm{M}$ TMR-dextran at DIV 13-15. A, B) Representative images of TMRdextran-loaded nerve terminals of WT $(A)$ and $K O(B)$ neurons. Scale bar $=30 \mu \mathrm{m}$. C) Mean TMR-dextran uptake as a proportion of total WT uptake \pm SEM. D-F) Fmr1 KO or WT neurons were challenged with a $40 \mathrm{~Hz} 10 \mathrm{~s}$ AP train in the presence of $10 \mathrm{mg} / \mathrm{ml} \mathrm{HRP}$ at DIV 13-15. D, E) Representative images of HRPlabelled endosomes and SVs in WT (D) and KO (E) nerve terminals. Black arrows indicate HRP endosomes, scale bar $=200 \mathrm{~nm}$. F) Mean HRP-labelled endosomes per nerve terminal \pm SEM. For statistical information see Table S1. 


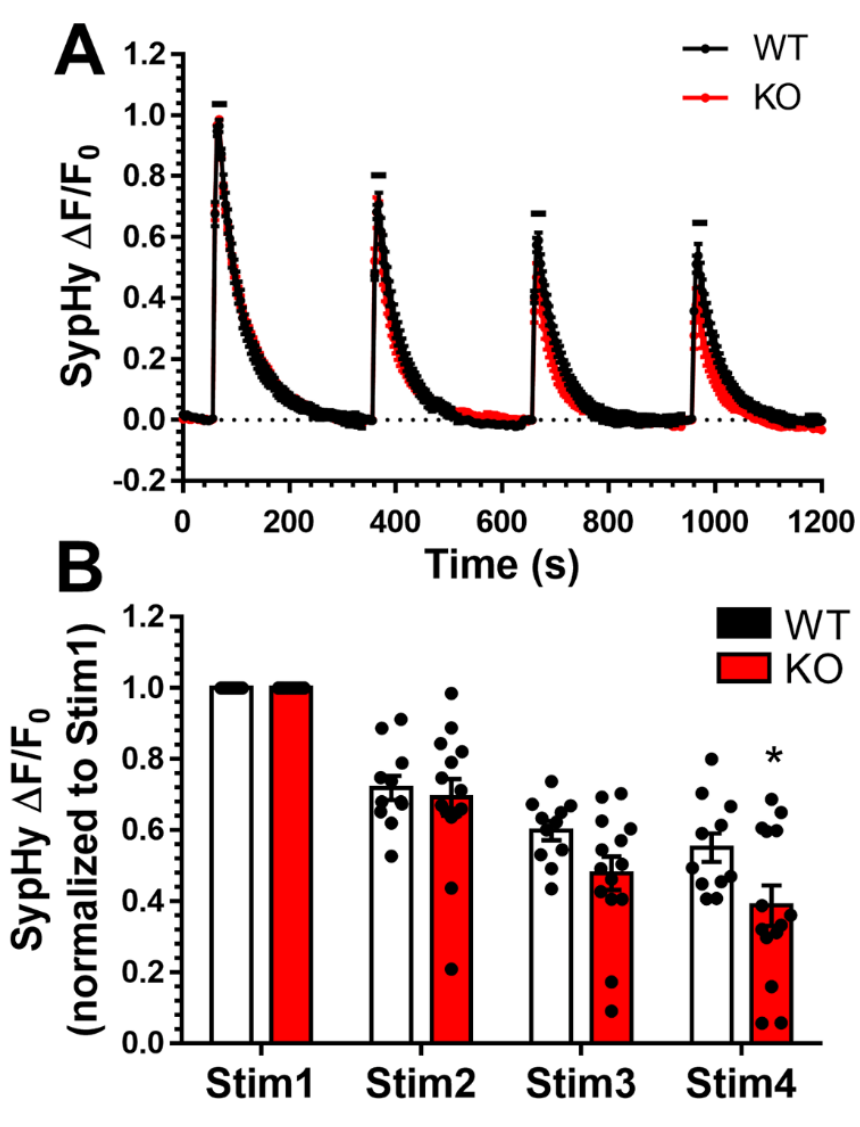

Figure 3. Fmr1 KO neurons display decreased presynaptic performance.

Hippocampal neurons from either Fmr1 KO or WT littermate controls were transfected with sypHy on DIV 7 and imaged DIV 13-15. Transfected neurons were stimulated four times with $40 \mathrm{~Hz} 10 \mathrm{~s}$ at $5 \mathrm{~min}$ intervals, before exposure to $\mathrm{NH}_{4} \mathrm{Cl}$. A) Mean traces displaying the average sypHy response of WT (black) and $\mathrm{KO}$ (red) neurons $\pm \mathrm{SEM}$. Traces are $\Delta F / F_{0}$ and normalised to the sypHy peak response to the first stimulus. Bar indicates stimulation period. B) Mean sypHy peak heights for each $40 \mathrm{~Hz} 10 \mathrm{~s}$ stimulation, normalised to the first \pm SEM. For statistical information see Table S1. 

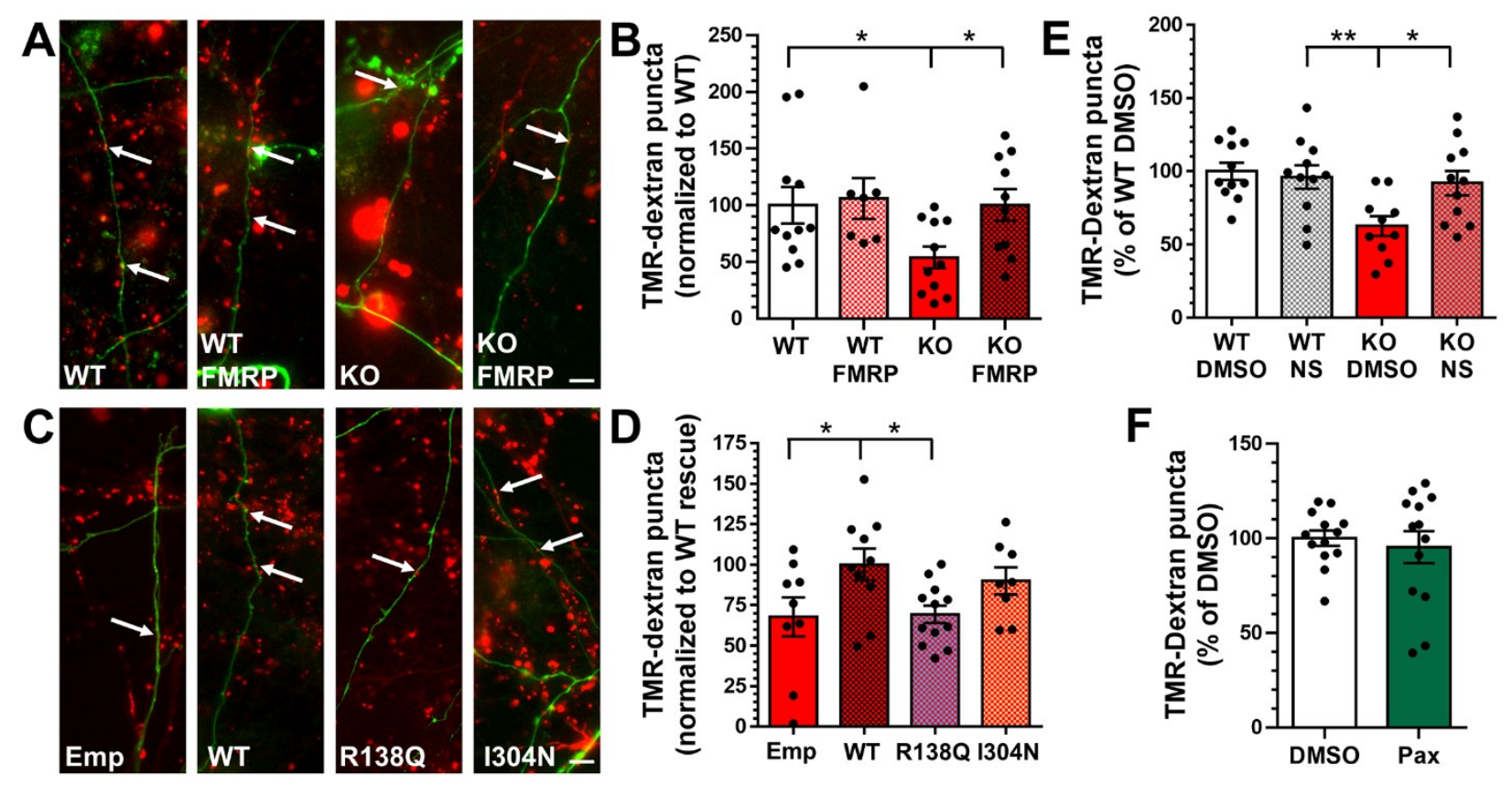

Figure 4. BK channel activation corrects ADBE defects in Fmr1 KO neurons

A-D) Hippocampal neurons from either Fmr1 KO or WT littermate controls were transfected with mCerulean (empty) or mCerulean and either GFP-FMRPwT $(\mathrm{WT})$, GFP-FMRP $\mathrm{R}_{138 \mathrm{Q}}(\mathrm{R} 138 \mathrm{C}$ ) or GFPFMRP $_{1304 \mathrm{~N}}($ I304N) 3 days prior to imaging. On DIV 13-15, neurons were challenged with a $40 \mathrm{~Hz} 10 \mathrm{~s}$ stimulus in the presence of $50 \mu \mathrm{M}$ TMR-dextran. A, C) Representative images of TMR-dextran uptake (red) in axons transfected with empty or FMRP vectors (green), arrows indicate TMR-dextran puncta. Scale bar $=10 \mu \mathrm{m}$. B, D) Mean TMR-dextran uptake per $100 \mu \mathrm{m}$ of transfected axon normalised to either B) WT control or D) FMRPwT control (both \pm SEM). E) Hippocampal neurons from either Fmr1 KO or WT littermate controls were incubated with DMSO or $10 \mu \mathrm{M}$ NS 11021 (NS), and $50 \mu \mathrm{M}$ TMR-dextran for 2 min prior to a $40 \mathrm{~Hz} 10 \mathrm{~s}$ stimulus on DIV 13-15. Mean TMR-dextran uptake as a proportion of total WT uptake \pm SEM. F) WT hippocampal neurons were incubated with DMSO or $10 \mu \mathrm{M}$ Paxilline (Pax) and $50 \mu \mathrm{M}$ TMR-dextran for 2 min prior to a $40 \mathrm{~Hz} 10 \mathrm{~s}$ stimulus on DIV 13-15. Mean TMR-dextran uptake as a proportion of total WT uptake \pm SEM. For statistical information see Table S1. 


\section{MATERIALS AND METHODS}

\section{Materials}

Unless otherwise specified, all cell culture reagents were obtained from ThermoFisher Scientific (Paisley, UK). Foetal bovine serum was from Biosera (Nuaille, France). Papain was obtained from Worthington Biochemical (Lakewood, NJ, USA). All other reagents were obtained from Sigma-Aldrich (Poole, UK). Rabbit anti-SV2A was obtained from Abcam (Cambridge, UK; ab32942; RRID: AB_778192). Anti-rabbit Alexa Fluor 488 was obtained from Invitrogen (Paisley, UK; A11008; RRID AB_143165).

\section{Animals}

Animal work was performed in accordance with the UK Animal (Scientific Procedures) Act 1986, under Project and Personal Licence authority and was approved by the Animal Welfare and Ethical Review Body at the University of Edinburgh (Home Office project licence - 7008878). Specifically, all animals were killed by schedule 1 procedures in accordance with UK Home Office Guidelines; adults were killed by exposure to $\mathrm{CO}_{2}$ followed by decapitation, whereas embryos were killed by decapitation followed by destruction of the brain. Heterozygous LE-Fmr1 $1^{\mathrm{m} 1 / \mathrm{PWC}}$ female rats (Asiminas et al., 2019) were mated with WT males to produce either Fmr1 KO or WT males. Male embryos were taken at e18.5-e19.5 for hippocampal dissection.

\section{DNA constructs}

Site-directed mutagenesis was used to introduce single base pair mutations into the hEGFP-FMRP isoform 1 plasmid (Khayachi et al., 2018) obtained from Dr B. Bardoni (INSERM, IPMC CNRS). hEGFPFMRP $_{\mathrm{R} 138 \mathrm{Q}}$ and $\mathrm{hEGFP-FMRP} \mathrm{P}_{1304 \mathrm{~N}}$ were generated using site-targeted mutagenesis (R138Q forward primer GTGCCAGAAGACTTACAGCAAATGTGTGCCAAA; reverse primer TTTGGCACACATTTGCTGTAAGTCTTCTGGCAC: I304N forward primer AAAAATGGAAAGCTGAATCAGGAGATTGTGGAC; reverse GTCCACAATCTCCTGAITCAGCTTTCCATTTTT (mutated bases underlined). The base change was confirmed by Source Bioscience Sanger Sequencing (Glasgow, UK). The mCerN1 (empty vector) was constructed as previously described (Cheung et al., 2010). Synaptophysin-pHluorin (sypHy) (Granseth et al., 2006) was provided by Prof. L. Lagnado (University of Sussex, UK).

\section{Primary hippocampal neuronal cultures and transfection}

Hippocampi from each embryo were processed separately to avoid contamination across genotypes. Dissociated primary hippocampal cultures were prepared from embryos as previously described (Zhang et al., 2015). Briefly, isolated hippocampi were digested in a $10 \mathrm{U} / \mathrm{mL}$ papain solution (Worthington Biochemical, LK003178) at $37^{\circ} \mathrm{C}$ for $20 \mathrm{~min}$. The papain was then neutralised using DMEM F12 (ThermoFisher Scientific, 21331-020) supplemented with $10 \%$ Foetal bovine serum (BioSera, S1810500 ) and $1 \%$ penicillin/streptomycin (ThermoFisher Scientific, 15140-122). Cells were triturated to form a single cell suspension and plated at $5 \times 10^{4}$ cells (with the exception of single cell TMR-dextran uptake experiments, $2.5 \times 10^{4}$ cells) per coverslip on laminin (10 $\mu \mathrm{g} / \mathrm{mL}$; Sigma Aldrich, L2020) and poly-D-lysine (Sigma Aldrich, P7886) coated $25 \mathrm{~mm}$ glass coverslips (VWR International Ltd, Lutterworth, UK). Cultures were maintained in Neurobasal media (ThermoFisher Scientific, 21103-049) supplemented with $2 \%$ B-27 (ThermoFisher Scientific, 17504-044), 0.5 mM L-glutamine (ThermoFisher Scientific, 25030-024) and 1\% penicillin/streptomycin. After 2-3 days in vitro (DIV), $1 \mu \mathrm{M}$ of cytosine arabinofuranoside (Sigma Aldrich, C1768) was added to each well to inhibit glial proliferation. Hippocampal neurons were transfected with sypHy at DIV 7 using Lipofectamine 2000 (ThermoFisher Scientific, 11668027) as per manufacturer's instructions and imaged at DIV 13-15. For single-cell dextran experiments, neurons were transfected with mCerN1 or hEGFP-FMRP constructs using Lipofectamine 20003 days prior to imaging at DIV 13-15.

\section{Fluorescence imaging of sypHy}


SypHy-transfected neurons were visualised at $500 \mathrm{~nm}$ band pass excitation with a $515 \mathrm{~nm}$ dichroic filter and a long-pass $>520 \mathrm{~nm}$ emission filter on a Zeiss Axio Observer D1 inverted epifluorescence microscope (Cambridge, UK). Images were captured using an AxioCam 506 mono camera (Zeiss) with a Zeiss EC Plan Neofluar 40x/1.30 oil immersion objective. Image acquisition was performed using Zen Pro software (Zeiss). Hippocampal cultures were mounted in a Warner Instruments (Hamden, CT, USA) imaging chamber with embedded parallel platinum wires (RC-21BRFS) and challenged with field stimulation using a Digitimer LTD MultiStim system-D330 stimulator (current output $100 \mathrm{~mA}$, current width $1 \mathrm{~ms}$ ) either at $10 \mathrm{~Hz}$ for $30 \mathrm{~s}, 40 \mathrm{~Hz}$ for $10 \mathrm{~s}$ or $4 \mathrm{X} 40 \mathrm{~Hz}$ for $10 \mathrm{~s}$ with $5 \mathrm{~min}$ intervals between trains. Imaging time courses were acquired at $4 \mathrm{~s}$ intervals while undergoing constant perfusion with imaging buffer (119 mM NaCl, $2.5 \mathrm{mM} \mathrm{KCl}, 2 \mathrm{mM} \mathrm{CaCl}$, $2 \mathrm{mM} \mathrm{MgCl}_{2}, 25 \mathrm{mM}$ HEPES, $30 \mathrm{mM}$ glucose at pH 7.4, supplemented with $10 \mu \mathrm{M}$ 6-cyano-7-nitroquinoxaline-2,3-dione (Abcam, Cambridge, UK, ab120271) and $50 \mu \mathrm{M}$ DL-2-Amino-5-phosphonopentanoic acid (Abcam, Cambridge, UK, ab120044). $\mathrm{NH}_{4} \mathrm{Cl}$ alkaline buffer $\left(50 \mathrm{mM} \mathrm{NH}_{4} \mathrm{Cl}\right.$ substituted for $50 \mathrm{mM} \mathrm{NaCl}$ ) was used to reveal the maximal pHluorin response.

\section{Measurement of functional SV pool size}

The size of the different functional SV pools in hippocampal neurons were measured by stimulating sypHy-transfected neurons for increasing durations in the presence of $1 \mu \mathrm{M}$ bafilomycin $\mathrm{A} 1$ (Cayman Chemical Company, Ann Arbor Michigan, USA, 11038). The RRP was mobilised by 40 APs (20 Hz), and $10 \mathrm{~s}$ later the remainder of the recycling pool mobilised with a second challenge of $1600 \mathrm{APs}(20 \mathrm{~Hz})$. The resting pool was revealed by application of $\mathrm{NH}_{4} \mathrm{Cl}$ buffer.

\section{Analysis of sypHy fluorescence traces}

Time traces were analysed using the FIJI distribution of Image $\mathrm{J}$ (National Institutes of Health). Images were aligned using the Rigid body model of the StackReg plugin (https://imagej.net/StackReg) (Thevenaz et al., 1998). Nerve terminal fluorescence was measured using the Time Series Analyser plugin (https://imagej.nih.gov/ij/plugins/time-series.html) (Balaji and Ryan, 2007). Regions of interest (ROIs) 5 pixels in diameter were placed over nerve terminals that responded to the electrical stimulus. A response trace was calculated for each cell by averaging the individual traces from each selected ROI.

Fluorescence decay time constants (tau, $\mathrm{T}, \mathrm{S}$ ) were calculated by fitting a monoexponential decay curve to data from the time point after the end of electrical stimulation.

\section{Fluorescence imaging of TMR-dextran uptake}

TMR-dextran (ThermoFisher Scientific, D1842) uptake was performed as described previously (Nicholson-Fish et al., 2015). Neurons were mounted on a Zeiss Axio Observer D1 microscope as described above before challenging with 400 action potentials $(40 \mathrm{~Hz})$ in the presence of $50 \mu \mathrm{M}$ of TMRdextran $(40,000 \mathrm{MW})$ in imaging buffer. Where the experiment was performed with BK channel modulators, neurons were incubated in $50 \mu \mathrm{M}$ of TMR-dextran and either DMSO (Sigma Aldrich, D8418), $10 \mu \mathrm{M}$ NS 11021 (Bio-Techne Ltd, Abingdon, UK, 4788/10) or $10 \mu \mathrm{M}$ Paxilline (Bio-Techne Ltd,

Abingdon, UK, 2006/10) for $120 \mathrm{~s}$ prior to stimulation. The TMR-dextran solution was immediately washed away after stimulation terminated, and images were acquired using 556/25 nm excitation and $630 / 98 \mathrm{~nm}$ emission bandpass filters (Zeiss) while undergoing constant perfusion. Per coverslip of cells, 3-6 different fields of view were imaged. The TMR-dextran puncta in each image were quantified using the Analyze Particles plugin of Image J (NIH, https://imagej.nih.gov/ij/developer/api/ij/plugin/filter/ParticleAnalyzer.html) to select and count particles of $0.23-0.91 \mu \mathrm{m}^{2}$.

Where TMR-dextran uptake was performed on transfected cultures, WT and KO neurons were transfected 3 days prior to imaging with mCer-N1 plus either hEGFP-FMRPwT, hEGFP-FMRPI304N or hEGFP-FMRP $\mathrm{R}_{138 \mathrm{Q}}$ or mCer-N1 alone. Transfected axons were visualised on DIV 13-15 at both $430 \mathrm{~nm}$ 
and $500 \mathrm{~nm}$ excitation (long-pass emission filter $>520 \mathrm{~nm}$ ) to ensure co-transfection. Images of transfected neurons and of TMR-dextran were acquired using 470/27 nm and 556/25 nm double band pass filters and emission filters 512/30 nm and 630/98 nm respectively (Zeiss). Per coverslip of cells, 2-8 neurons were imaged. Axon length was calculated using the Simple Neurite Tracer plugin of Image $\mathrm{J}$ (NIH, https://imagej.net/SNT). TMR-dextran puncta $\left(0.23-0.91 \mu \mathrm{m}^{2}\right)$ were counted along transfected axons with the final value normalised for axon length. For all experiments, for each condition, at least one unstimulated coverslip was imaged to correct for the background level of TMR-dextran uptake.

\section{Immunofluorescence staining}

Immunofluorescence staining was performed as previously described (Nicholson-Fish et al., 2015). Briefly, hippocampal neurons were fixed with $4 \%$ paraformaldehyde (Sigma Aldrich, 47608) in PBS for 15 min. Excess paraformaldehyde was quenched with $50 \mathrm{mM} \mathrm{NH}_{4} \mathrm{Cl}$ in PBS. Cells were then permeabilized in $1 \%$ bovine serum albumin (BSA; Roche Diagnostics $\mathrm{GmbH}$, Germany, 10735078001) in PBS-Triton $0.1 \%$ solution for $5 \mathrm{~min}$ and blocked in $1 \% \mathrm{BSA}$ in PBS at room temperature for $1 \mathrm{~h}$. After blocking, cells were incubated in rabbit anti-SV2A (1:200 dilution) for $1 \mathrm{~h}$, after which the cultures were washed with PBS and incubated with fluorescently conjugated secondary antibodies (anti-rabbit Alexa Fluor 488; 1:1000 dilution) for $1 \mathrm{hr}$. The coverslips were mounted on slides for imaging with FluorSave (Millipore, Darmstadt, Germany, 345789). SV2A puncta were visualised at $500 \mathrm{~nm}$ band pass excitation with a $515 \mathrm{~nm}$ dichroic filter and a long-pass $>520 \mathrm{~nm}$ emission filter on a Zeiss Axio Observer D1 inverted epifluorescence microscope (Cambridge, UK). Images were captured using an AxioCam 506 mono camera (Zeiss) with a Zeiss EC Plan Neofluar 40x/1.30 oil immersion objective. SV2A puncta in each image were quantified using the Analyze Particles plugin of Image $\mathrm{J}$ to select and count particles of $0.23-3.18 \mu \mathrm{m}^{2}$.

\section{HRP uptake}

Hippocampal cultures were mounted in the RC-21BRFS stimulation chamber and challenged with 400 action potentials $(40 \mathrm{~Hz}$ ) in the presence of $10 \mathrm{mg} / \mathrm{ml} \mathrm{HRP} \mathrm{(Sigma} \mathrm{Aldrich,} \mathrm{P8250)} \mathrm{supplemented} \mathrm{imaging}$ buffer. Immediately following the end of stimulation, cultures were washed in imaging buffer to remove non-internalised HRP and fixed with a solution of $2 \%$ glutaraldehyde (Electron Microscopy Sciences, Hatfield, USA, 16019) in phosphate buffered saline. After washing in $0.1 \mathrm{M}$ Tris buffer, HRP was developed with $0.1 \%$ 3,3'-diaminobenzidine (Fluka Chemica, Gillingham, UK, 22204001) and $0.2 \%$ v/v hydrogen peroxide (Honeywell, Muskegon, USA, 216763) in Tris buffer. After further washing in Tris buffer, cultures were then stained with $1 \%$ osmium tetroxide (TAAB laboratory and microscopy, Aldermaston, UK, O015/1) for $30 \mathrm{~min}$. Samples were then dehydrated using an ethanol series and polypropylene oxide (Electron Microscopy Sciences, Hatfield, USA, 20411) and embedded using Durcupan resin (Sigma Aldrich, 44610). Samples were sectioned, mounted on grids, and viewed using an FEI Tecnai 12 transmission electron microscope (Oregon, USA). Intracellular structures that were $<61 \mathrm{~nm}$ in diameter were arbitrarily designated to be SVs, whereas larger structures were considered endosomes. The endosome area was obtained by tracing the circumference using the freehand selections tool in Image $J$ and measuring the resulting area. Typically, 30 fields of view were acquired for one coverslip of cells. The average number of HRP-labelled endosomes and SVs per nerve terminal was calculated for each coverslip and represents the experimental $n$.

\section{Quantification and data analysis}

Microsoft Excel (Microsoft, Washington, USA) and Prism 8 software (GraphPad software Inc., San Diego USA) were used for data processing and analysis. The experimenter was blinded during both the acquisition and analysis of data. For all figures, results are presented with error bars as \pm SEM, and the $n$ for each condition represents the number of coverslips imaged. For all assays, cells were obtained from at least two cultures $(\mathrm{N})$, with independent preparations from at least three individual embryos. 


\section{ACKNOWLEDGEMENTS}

Work is supported by the Simons Foundation (529508), The RS McDonald fund and a College of Medicine and Veterinary Medicine studentship. We thank Jennifer Darnell for expert advice and Steven Mitchell for excellent technical assistance. The authors declare no competing financial interests.

\section{AUTHOR CONTRIBUTIONS}

Conceptualization, PCK, MAC; Methodology, KB, MAC; Data analysis, KB; Investigation, KB, MAC; Resources, PCK; Writing - All; Funding Acquisition, PCK, MAC.

\section{REFERENCE LIST}

Alabi, A.A., and R.W. Tsien. 2012. Synaptic vesicle pools and dynamics. Cold Spring Harb Perspect.Biol. 4:a013680. doi: 10.1101/cshperspect.a013680 [doi].

Asiminas, A., A.D. Jackson, S.R. Louros, S.M. Till, T. Spano, O. Dando, M.F. Bear, S. Chattarji, G.E. Hardingham, E.K. Osterweil, D.J.A. Wyllie, E.R. Wood, and P.C. Kind. 2019. Sustained correction of associative learning deficits after brief, early treatment in a rat model of Fragile $X$ Syndrome.

Sci.Transl.Med. 11:10.1126/scitransImed.aao0498. doi: eaao0498 [pii].

Atluri, P.P., and T.A. Ryan. 2006. The kinetics of synaptic vesicle reacidification at hippocampal nerve terminals. J.Neurosci. 26:2313-2320. doi: 26/8/2313 [pii].

Balaji, J., and T.A. Ryan. 2007. Single-vesicle imaging reveals that synaptic vesicle exocytosis and endocytosis are coupled by a single stochastic mode. Proc.Natl.Acad.Sci.U.S.A. 104:20576-20581. doi: 0707574105 [pii].

Booker, S.A., A.P.F. Domanski, O.R. Dando, A.D. Jackson, J.T.R. Isaac, G.E. Hardingham, D.J.A. Wyllie, and P.C. Kind. 2019. Altered dendritic spine function and integration in a mouse model of fragile $X$ syndrome. Nat.Commun. 10:4813. doi: 10.1038/s41467-019-11891-6 [doi].

Brown, M.R., J. Kronengold, V.R. Gazula, Y. Chen, J.G. Strumbos, F.J. Sigworth, D. Navaratnam, and L.K. Kaczmarek. 2010. Fragile $X$ mental retardation protein controls gating of the sodium-activated potassium channel Slack. Nat.Neurosci. 13:819-821. doi: 10.1038/nn.2563; 10.1038/nn.2563.

Chanaday, N.L., M.A. Cousin, I. Milosevic, S. Watanabe, and J.R. Morgan. 2019. The Synaptic Vesicle Cycle Revisited: New Insights into the Modes and Mechanisms. J.Neurosci. 39:8209-8216. doi: 10.1523/JNEUROSCI.1158-19.2019 [doi].

Cheung, G., O.J. Jupp, and M.A. Cousin. 2010. Activity-dependent bulk endocytosis and clathrindependent endocytosis replenish specific synaptic vesicle pools in central nerve terminals. J.Neurosci. 30:8151-8161. doi: 10.1523/JNEUROSCI.0293-10.2010 [doi].

Clayton, E.L., G.J. Evans, and M.A. Cousin. 2008. Bulk synaptic vesicle endocytosis is rapidly triggered during strong stimulation. J.Neurosci. 28:6627-6632. doi: 10.1523/JNEUROSCI.1445-08.2008; 10.1523/JNEUROSCI.1445-08.2008.

Collins, S.C., S.M. Bray, J.A. Suhl, D.J. Cutler, B. Coffee, M.E. Zwick, and S.T. Warren. 2010. Identification of novel FMR1 variants by massively parallel sequencing in developmentally delayed males. Am.J.Med.Genet.A. 152A:2512-2520. doi: 10.1002/ajmg.a.33626 [doi]. 
Cousin, M.A., and P.J. Robinson. 2000. Ca(2+) influx inhibits dynamin and arrests synaptic vesicle endocytosis at the active zone. J.Neurosci. 20:949-957.

Darnell, J.C., and E. Klann. 2013. The translation of translational control by FMRP: therapeutic targets for FXS. Nat.Neurosci. doi: 10.1038/nn.3379; 10.1038/nn.3379.

Das Sharma, S., R. Pal, B.K. Reddy, B.T. Selvaraj, N. Raj, K.K. Samaga, D.J. Srinivasan, L. Ornelas, D. Sareen, M.R. Livesey, G.J. Bassell, C.N. Svendsen, P.C. Kind, S. Chandran, S. Chattarji, and D.J.A. Wyllie. 2020. Cortical neurons derived from human pluripotent stem cells lacking FMRP display altered spontaneous firing patterns. Mol.Autism. 11:52. doi: 10.1186/s13229-020-00351-4 [doi].

De Boulle, K., A.J. Verkerk, E. Reyniers, L. Vits, J. Hendrickx, B. Van Roy, F. Van den Bos, E. de Graaff, B.A. Oostra, and P.J. Willems. 1993. A point mutation in the FMR-1 gene associated with fragile $X$ mental retardation. Nat.Genet. 3:31-35. doi: 10.1038/ng0193-31 [doi].

Deng, P.Y., and V.A. Klyachko. 2016. Genetic upregulation of BK channel activity normalizes multiple synaptic and circuit defects in a mouse model of fragile X syndrome. J.Physiol. 594:83-97. doi: 10.1113/JP271031 [doi].

Deng, P.Y., Z. Rotman, J.A. Blundon, Y. Cho, J. Cui, V. Cavalli, S.S. Zakharenko, and V.A. Klyachko. 2013. FMRP regulates neurotransmitter release and synaptic information transmission by modulating action potential duration via BK channels. Neuron. 77:696-711. doi: 10.1016/j.neuron.2012.12.018; 10.1016/j.neuron.2012.12.018.

Deng, P.Y., D. Sojka, and V.A. Klyachko. 2011. Abnormal presynaptic short-term plasticity and information processing in a mouse model of fragile $X$ syndrome. J.Neurosci. 31:10971-10982. doi: 10.1523/JNEUROSCI.2021-11.2011; 10.1523/JNEUROSCI.2021-11.2011.

Diaz, J., C. Scheiner, and E. Leon. 2018. Presentation of a recurrent FMR1 missense mutation (R138Q) in an affected female. Translational Science of Rare Diseases. 3:139-144.

Feng, Y., D. Absher, D.E. Eberhart, V. Brown, H.E. Malter, and S.T. Warren. 1997. FMRP associates with polyribosomes as an mRNP, and the I304N mutation of severe fragile $X$ syndrome abolishes this association. Mol.Cell. 1:109-118. doi: S1097-2765(00)80012-X [pii].

Ferron, L. 2016. Fragile $X$ mental retardation protein controls ion channel expression and activity. J.Physiol. 594:5861-5867. doi: 10.1113/JP270675 [doi].

Ferron, L., M. Nieto-Rostro, J.S. Cassidy, and A.C. Dolphin. 2014. Fragile X mental retardation protein controls synaptic vesicle exocytosis by modulating N-type calcium channel density. Nat.Commun. 5:3628. doi: $10.1038 /$ ncomms4628 [doi].

Gamache, T.R., Y. Araki, and R.L. Huganir. 2020. Twenty Years of SynGAP Research: From Synapses to Cognition. J.Neurosci. 40:1596-1605. doi: 10.1523/JNEUROSCI.0420-19.2020 [doi].

Granseth, B., B. Odermatt, S.J. Royle, and L. Lagnado. 2006. Clathrin-mediated endocytosis is the dominant mechanism of vesicle retrieval at hippocampal synapses. Neuron. 51:773-786. doi: S08966273(06)00675-1 [pii].

Hebert, B., S. Pietropaolo, S. Meme, B. Laudier, A. Laugeray, N. Doisne, A. Quartier, S. Lefeuvre, L. Got, D. Cahard, F. Laumonnier, W.E. Crusio, J. Pichon, A. Menuet, O. Perche, and S. Briault. 2014. Rescue of fragile $\mathrm{X}$ syndrome phenotypes in Fmr1 KO mice by a BKCa channel opener molecule. Orphanet J.Rare Dis. 9:124. doi: 10.1186/s13023-014-0124-6 [doi]. 
Khayachi, A., C. Gwizdek, G. Poupon, D. Alcor, M. Chafai, F. Cassé, T. Maurin, M. Prieto, A. Folci, F. De Graeve, S. Castagnola, R. Gautier, L. Schorova, C. Loriol, M. Pronot, F. Besse, F. Brau, E. Deval, B. Bardoni, and S. Martin. 2018. Sumoylation regulates FMRP-mediated dendritic spine elimination and maturation. Nat.Commun. 9:757. doi: 10.1038/s41467-018-03222-y [doi].

Klemmer, P., R.M. Meredith, C.D. Holmgren, O.I. Klychnikov, J. Stahl-Zeng, M. Loos, R.C. van der Schors, J. Wortel, H. de Wit, S. Spijker, D.C. Rotaru, H.D. Mansvelder, A.B. Smit, and K.W. Li. 2011. Proteomics, ultrastructure, and physiology of hippocampal synapses in a fragile $X$ syndrome mouse model reveal presynaptic phenotype. J.Biol.Chem. 286:25495-25504. doi: 10.1074/jbc.M110.210260; 10.1074/jbc.M110.210260.

Kokotos, A.C., J. Peltier, E.C. Davenport, M. Trost, and M.A. Cousin. 2018. Activity-dependent bulk endocytosis proteome reveals a key presynaptic role for the monomeric GTPase Rab11.

Proc.Natl.Acad.Sci.U.S.A. 115:E10177-E10186. doi: 10.1073/pnas.1809189115 [doi].

Kshatri, A., A. Cerrada, R. Gimeno, D. Bartolome-Martin, P. Rojas, and T. Giraldez. 2020. Differential regulation of BK channels by fragile $\mathrm{X}$ mental retardation protein. J.Gen.Physiol.

152:10.1085/jgp.201912502. doi: e201912502 [pii].

Leitz, J., and E.T. Kavalali. 2016. Ca2+ Dependence of Synaptic Vesicle Endocytosis. Neuroscientist. 22:464-476. doi: 10.1177/1073858415588265 [doi].

Lopez-Murcia, F.J., S.J. Royle, and A. Llobet. 2014. Presynaptic clathrin levels are a limiting factor for synaptic transmission. J.Neurosci. 34:8618-8629. doi: 10.1523/JNEUROSCI.5081-13.2014 [doi].

Mefford, H.C., M.L. Batshaw, and E.P. Hoffman. 2012. Genomics, intellectual disability, and autism. N.Engl.J.Med. 366:733-743. doi: 10.1056/NEJMra1114194 [doi].

Myrick, L.K., P.Y. Deng, H. Hashimoto, Y.M. Oh, Y. Cho, M.J. Poidevin, J.A. Suhl, J. Visootsak, V. Cavalli, P. Jin, X. Cheng, S.T. Warren, and V.A. Klyachko. 2015. Independent role for presynaptic FMRP revealed by an FMR1 missense mutation associated with intellectual disability and seizures.

Proc.NatI.Acad.Sci.U.S.A. 112:949-956. doi: 10.1073/pnas.1423094112 [doi].

Nicholson-Fish, J.C., A.C. Kokotos, T.H. Gillingwater, K.J. Smillie, and M.A. Cousin. 2015. VAMP4 Is an Essential Cargo Molecule for Activity-Dependent Bulk Endocytosis. Neuron. 88:973-984. doi: S08966273(15)00937-X [pii].

Richards, D.A., C. Guatimosim, and W.J. Betz. 2000. Two endocytic recycling routes selectively fill two vesicle pools in frog motor nerve terminals. Neuron. 27:551-559. doi: S0896-6273(00)00065-9 [pii].

Sankaranarayanan, S., and T.A. Ryan. 2001. Calcium accelerates endocytosis of vSNAREs at hippocampal synapses. Nat.Neurosci. 4:129-136. doi: 10.1038/83949 [doi].

Sitzmann, A.F., R.T. Hagelstrom, F. Tassone, R.J. Hagerman, and M.G. Butler. 2018. Rare FMR1 gene mutations causing fragile X syndrome: A review. Am.J.Med.Genet.A. 176:11-18. doi:

10.1002/ajmg.a.38504 [doi].

Soykan, T., N. Kaempf, T. Sakaba, D. Vollweiter, F. Goerdeler, D. Puchkov, N.L. Kononenko, and V. Haucke. 2017. Synaptic Vesicle Endocytosis Occurs on Multiple Timescales and Is Mediated by ForminDependent Actin Assembly. Neuron. 93:854-866.e4. doi: S0896-6273(17)30095-8 [pii].

Thevenaz, P., U.E. Ruttimann, and M. Unser. 1998. A pyramid approach to subpixel registration based on intensity. IEEE Trans.Image Process. 7:27-41. doi: 10.1109/83.650848 [doi]. 
von Gersdorff, H., and G. Matthews. 1994. Inhibition of endocytosis by elevated internal calcium in a synaptic terminal. Nature. 370:652-655. doi: 10.1038/370652a0 [doi].

Wang, X.S., C.Z. Peng, W.J. Cai, J. Xia, D. Jin, Y. Dai, X.G. Luo, V.A. Klyachko, and P.Y. Deng. 2014. Activity-dependent regulation of release probability at excitatory hippocampal synapses: a crucial role of fragile X mental retardation protein in neurotransmission. Eur.J.Neurosci. 39:1602-1612. doi:

10.1111/ejn.12546 [doi].

Watanabe, S., B.R. Rost, M. Camacho-Perez, M.W. Davis, B. Sohl-Kielczynski, C. Rosenmund, and E.M. Jorgensen. 2013. Ultrafast endocytosis at mouse hippocampal synapses. Nature. 504:242-247. doi: 10.1038/nature12809 [doi].

Wu, X.S., and L.G. Wu. 2014. The yin and yang of calcium effects on synaptic vesicle endocytosis. J.Neurosci. 34:2652-2659. doi: 10.1523/JNEUROSCI.3582-13.2014 [doi].

Zhang, N., S.L. Gordon, M.J. Fritsch, N. Esoof, D.G. Campbell, R. Gourlay, S. Velupillai, T. Macartney, M. Peggie, D.M. van Aalten, M.A. Cousin, and D.R. Alessi. 2015. Phosphorylation of synaptic vesicle protein $2 \mathrm{~A}$ at Thr84 by casein kinase 1 family kinases controls the specific retrieval of synaptotagmin-1.

J.Neurosci. 35:2492-2507. doi: 10.1523/JNEUROSCI.4248-14.2015 [doi].

Zhang, Y., A. Bonnan, G. Bony, I. Ferezou, S. Pietropaolo, M. Ginger, N. Sans, J. Rossier, B. Oostra, G. LeMasson, and A. Frick. 2014. Dendritic channelopathies contribute to neocortical and sensory hyperexcitability in Fmr1(-/y) mice. Nat.Neurosci. 17:1701-1709. doi: 10.1038/nn.3864 [doi].

\section{ABBREVIATIONS}

SV - synaptic vesicle; FMRP - fragile X mental retardation protein; FXS fragile X syndrome; AP - action potential; ADBE - activity-dependent bulk endocytosis; BK - big potassium; RRP - readily releasable pool; ID - intellectual disability; HRP - horseradish peroxidase; KO - knockout; WT - wild-type; DIV - day in vitro; TMR - tetramethylrhodamine; $\mathrm{BSA}$ - bovine serum albumin; $\mathrm{ROI}$ - region of interest; $\mathrm{mCer}$ mCerulean; sypHy - synaptophysin-pHluorin. 
A

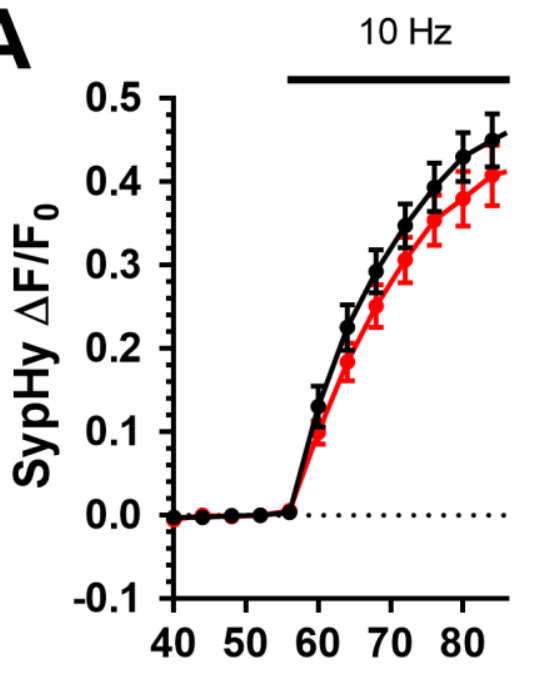

Time (s)

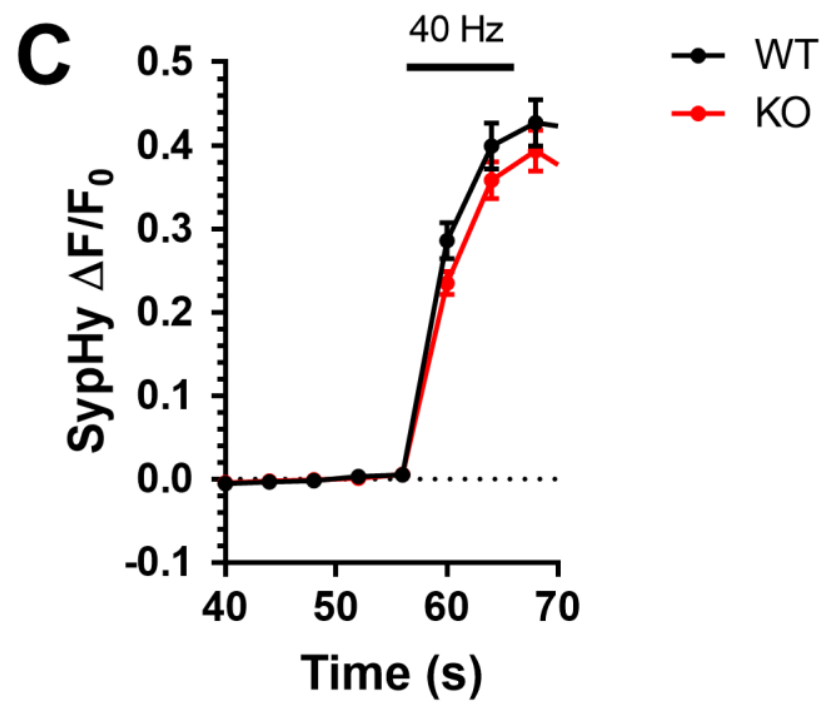

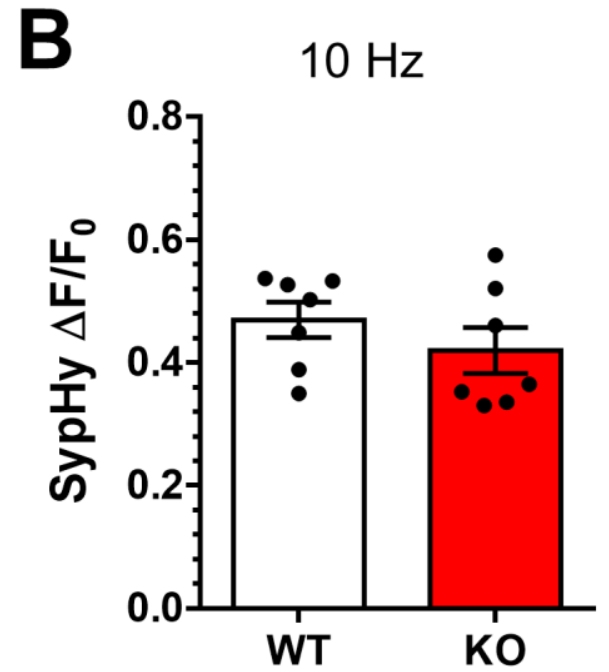

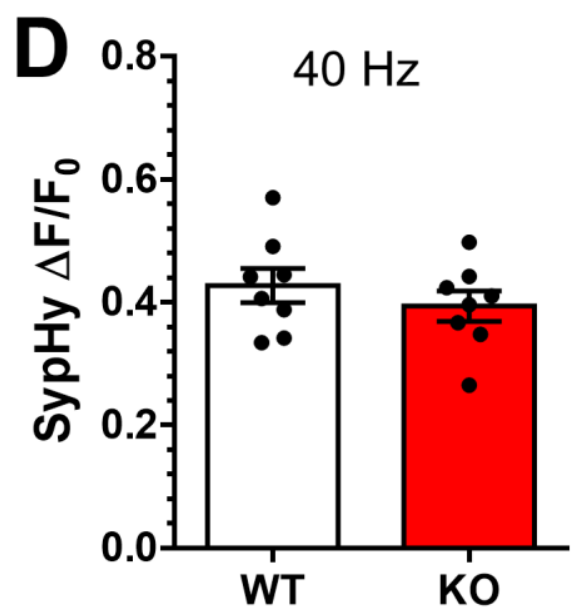

Figure S1. No difference in SV exocytosis across genotypes

Hippocampal neurons from either Fmr1 KO or WT littermate controls were transfected with sypHy on DIV 7 and imaged DIV 13-15 in the presence of $1 \mu \mathrm{M}$ bafilomycin A1. Neurons were challenged with a train of APs (either $10 \mathrm{~Hz} 30 \mathrm{~s}, \mathrm{~A}-\mathrm{B}$; or $40 \mathrm{~Hz} 10 \mathrm{~s}, \mathrm{C}-\mathrm{D}$ ) before exposure to $\mathrm{NH}_{4} \mathrm{Cl}$. A, C) Mean traces displaying the average sypHy response of WT (black) and KO (red) neurons in response to $10 \mathrm{~Hz}(\mathrm{~A})$ or $40 \mathrm{~Hz}(\mathrm{C})$ stimulation $\left(\triangle \mathrm{F} / \mathrm{F}_{0}\right.$ as a fraction of the total $\mathrm{SV}$ pool, revealed by $\left.\mathrm{NH}_{4} \mathrm{Cl}\right)$. Bar indicates stimulation period. $B, D)$ Mean sypHy peak heights during either $10 \mathrm{~Hz}(B)$ or $40 \mathrm{~Hz}(D)$ stimulation. A-D) All data shown \pm SEM. For statistical information see Table S1. 

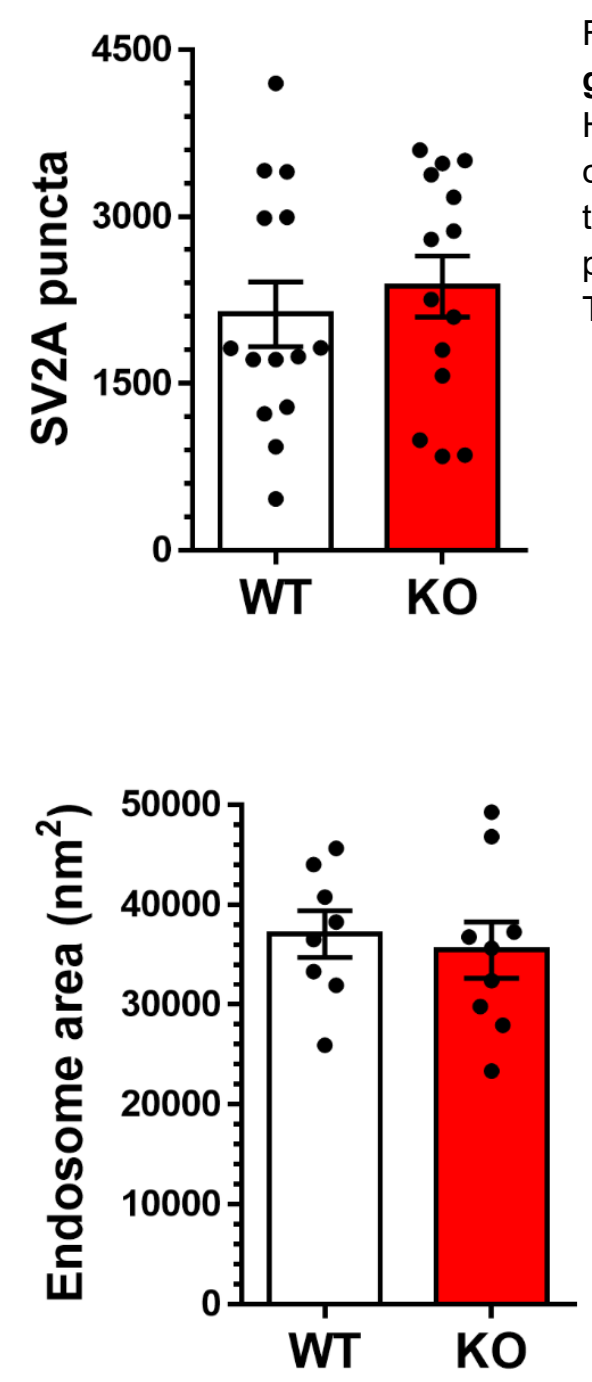

Figure S2. No difference in nerve terminal number between genotypes.

Hippocampal neurons derived from either Fmr1 KO or WT littermate controls were fixed and stained with anti-SV2A as a marker of nerve terminals. Bar graph represents the mean number of SV2A-stained puncta for both KO and WT \pm SEM, For statistical information see Table S1.
Figure S3. No difference in endosome size across genotypes.

Hippocampal neurons derived from either Fmr1 KO or WT littermate controls were challenged with a $40 \mathrm{~Hz} 10 \mathrm{~s}$ action potential train in the presence of $10 \mathrm{mg} / \mathrm{ml} \mathrm{HRP}$ at DIV 13-15. Mean HRP-labelled endosome areas in WT and KO \pm SEM. For statistical information see Table S1. 
bioRxiv preprint doi: https://doi.org/10.1101/2020.09.10.291062; this version posted February 17, 2021. The copyright holder for this preprint (which was not certified by peer review) is the author/funder, who has granted bioRxiv a license to display the preprint in perpetuity. It is made available under aCC-BY 4.0 International license.

\begin{tabular}{|c|c|c|c|c|c|c|}
\hline Figure 1 & Group & Mean \pm SEM & $\begin{array}{c}\mathrm{n}=\# \text { of } \\
\text { coverslips } / \mathrm{N}=\# \\
\text { of neuronal } \\
\text { preparations }\end{array}$ & Comparison & $\mathrm{P}$ & Statistical test \\
\hline \multirow{2}{*}{ Fig1C } & WT & $38.2 \pm 2.9 \%$ & $10 / 4$ & \multirow{2}{*}{ WT vs KO } & \multirow{2}{*}{0.6545} & \multirow{2}{*}{$\begin{array}{l}\text { Unpaired t- } \\
\text { test }\end{array}$} \\
\hline & $\mathrm{KO}$ & $40.4 \pm 3.7 \%$ & $10 / 4$ & & & \\
\hline \multirow{2}{*}{ Fig1D } & WT & $34.0 \pm 1.4 \mathrm{~s}$ & $10 / 4$ & \multirow{2}{*}{ WT vs KO } & \multirow{2}{*}{0.2076} & \multirow{2}{*}{$\begin{array}{l}\text { Unpaired t- } \\
\text { test }\end{array}$} \\
\hline & $\mathrm{KO}$ & $39.3 \pm 3.8 \mathrm{~s}$ & $10 / 4$ & & & \\
\hline \multirow{2}{*}{ Fig1F } & WT & $43.3 \pm 2.6 \%$ & $13 / 4$ & \multirow{2}{*}{ WT vs KO } & \multirow{2}{*}{0.4751} & \multirow{2}{*}{$\begin{array}{l}\text { Unpaired t- } \\
\text { test }\end{array}$} \\
\hline & $\mathrm{KO}$ & $39.9 \pm 3.9 \%$ & $13 / 4$ & & & \\
\hline \multirow{2}{*}{ Fig1G } & WT & $31.8 \pm 2.9 \mathrm{~s}$ & $13 / 4$ & \multirow{2}{*}{ WT vs KO } & \multirow{2}{*}{0.8382} & \multirow{2}{*}{$\begin{array}{l}\text { Unpaired t- } \\
\text { test }\end{array}$} \\
\hline & $\mathrm{KO}$ & $31.1 \pm 2.8 \mathrm{~s}$ & $13 / 4$ & & & \\
\hline \multirow{2}{*}{ Fig1l } & WT & $2.0 \pm 0.2 \mathrm{u}$ & $14 / 3$ & \multirow{2}{*}{ WT vs KO } & \multirow{2}{*}{0.8275} & \multirow{2}{*}{$\begin{array}{l}\text { Unpaired t- } \\
\text { test }\end{array}$} \\
\hline & $\mathrm{KO}$ & $2.0 \pm 0.2 u$ & $15 / 3$ & & & \\
\hline \multirow{2}{*}{ Fig1J } & WT & $12.0 \pm 0.9 \%$ & $14 / 3$ & \multirow{2}{*}{ WT vs KO } & \multirow{2}{*}{0.3715} & \multirow{2}{*}{$\begin{array}{l}\text { Unpaired t- } \\
\text { test }\end{array}$} \\
\hline & $\mathrm{KO}$ & $13.6 \pm 1.5 \%$ & $15 / 3$ & & & \\
\hline \multirow{2}{*}{ Fig1K } & WT & $46.5 \pm 2.8 \%$ & $14 / 3$ & \multirow{2}{*}{ WT vs KO } & \multirow{2}{*}{0.0299} & \multirow{2}{*}{$\begin{array}{l}\text { Unpaired t- } \\
\text { test }\end{array}$} \\
\hline & $\mathrm{KO}$ & $55.2 \pm 2.5 \%$ & $15 / 3$ & & & \\
\hline Figure 2 & Group & Mean \pm SEM & $\begin{array}{c}\mathrm{n}=\# \text { of } \\
\text { coverslips } / \mathrm{N}=\# \\
\text { of neuronal } \\
\text { preparations }\end{array}$ & Comparison & $\mathrm{P}$ & Statistical test \\
\hline \multirow{2}{*}{ Fig2C } & WT & $100.0 \pm 16.2 \%$ & $14 / 3$ & \multirow{2}{*}{ WT vs KO } & \multirow{2}{*}{0.0054} & Unpaired t- \\
\hline & $\mathrm{KO}$ & $76.0 \pm 5.2 \%$ & $14 / 3$ & & & test \\
\hline Fia2F & WT & $6.2 \pm 0.1$ endosomes & $8 / 3$ & WT vs KO & 00004 & Unpaired t- \\
\hline$r \mid g<r$ & $\mathrm{KO}$ & $4.8 \pm 0.3$ endosomes & $9 / 3$ & Wri vs nu & 0.0004 & test \\
\hline Figure 3 & Group & Mean \pm SEM & $\begin{array}{c}\mathrm{n}=\# \text { of cells or } \\
\text { coverslips } / \mathrm{N}=\# \\
\text { of neuronal } \\
\text { preparations }\end{array}$ & Comparison & $\mathrm{P}$ & Statistical test \\
\hline & WT Stim1 & $1.00 \pm 0.00 u$ & $\mathrm{n} 11 / 2$ & WT Stim1 vs KO & $>0.9999$ & \\
\hline & KO Stim1 & $1.00 \pm 0.00 u$ & n 14/2 & Stim1 & $>0.9999$ & \\
\hline & WT Stim2 & $0.72 \pm 0.03 u$ & n $11 / 2$ & WT Stim2 vs KO & $>09999$ & ANOVA with \\
\hline Fin $3 \mathrm{R}$ & KO Stim2 & $0.69 \pm 0.05 u$ & n 14/2 & Stim2 & $>0.9999$ & Bonferroni's \\
\hline TIgOD & WT Stim3 & $0.60 \pm 0.03 u$ & $\mathrm{n} 11 / 2$ & WT Stim3 vs KO & 01529 & multiple \\
\hline & KO Stim3 & $0.48 \pm 0.05 u$ & n 14/2 & Stim3 & 0.1529 & comparison \\
\hline & WT Stim4 & $0.55 \pm 0.04 u$ & $\mathrm{n} 11 / 2$ & WT Stim4 vs KO & 00217 & \\
\hline & KO Stim4 & $0.39 \pm 0.06 u$ & n 14/2 & Stim4 & 0.0211 & \\
\hline
\end{tabular}




\begin{tabular}{|c|c|c|c|c|c|c|}
\hline Figure 4 & Group & Mean \pm SEM & $\begin{array}{c}\mathrm{n}=\# \text { of } \\
\text { coverslips/ } \mathrm{N}=\# \\
\text { of neuronal } \\
\text { preparations }\end{array}$ & Comparison & $\mathrm{P}$ & Statistical test \\
\hline \multirow{4}{*}{ Fig4B } & WT & $100.0 \pm 16.2 \%$ & $11 / 4$ & \multirow{2}{*}{ WT vs KO } & \multirow{2}{*}{0.043} & \multirow{4}{*}{$\begin{array}{c}\text { 1-way } \\
\text { ANOVA with } \\
\text { Bonferroni's } \\
\text { multiple } \\
\text { comparisons } \\
\text { test }\end{array}$} \\
\hline & WT FMRP & $106.1 \pm 18.1 \%$ & $7 / 4$ & & & \\
\hline & $\mathrm{KO}$ & $54.1 \pm 9.6 \%$ & $11 / 4$ & \multirow[b]{2}{*}{ KO vs KO FMRP } & \multirow[b]{2}{*}{0.048} & \\
\hline & KO FMRP & $100.2 \pm 14.0 \%$ & $10 / 4$ & & & \\
\hline \multirow{4}{*}{ Fig4D } & Empty (Emp) & $67.8 \pm 12.1 \%$ & $8 / 3$ & WT vs empty & 0.043 & \multirow{4}{*}{$\begin{array}{c}\text { 1-way } \\
\text { ANOVA with } \\
\text { Dunnett's } \\
\text { multiple } \\
\text { comparison } \\
\text { test } \\
\end{array}$} \\
\hline & WT & $100.0 \pm 9.8 \%$ & $10 / 3$ & WT vs R138Q & 0.038 & \\
\hline & $\mathrm{R} 138 \mathrm{Q}$ & $69.4 \pm 5.4 \%$ & $8 / 3$ & \multirow[b]{2}{*}{ WT vs I304N } & \multirow[b]{2}{*}{0.788} & \\
\hline & $1304 N$ & $89.9 \pm 8.4 \%$ & $12 / 3$ & & & \\
\hline \multirow{4}{*}{ Fig4E } & WT DMSO & $100.0 \pm 5.9 \%$ & $11 / 3$ & $\begin{array}{l}\text { WT DMSO vs } \\
\text { KO DMSO }\end{array}$ & 0.0039 & \multirow{4}{*}{$\begin{array}{c}\text { 1-way } \\
\text { ANOVA with } \\
\text { Bonferroni's } \\
\text { multiple } \\
\text { comparisons } \\
\text { test }\end{array}$} \\
\hline & WT NS & $96.1 \pm 8.0 \%$ & $11 / 3$ & $\begin{array}{c}\text { KO DMSO vs } \\
\text { KO NS }\end{array}$ & 0.0322 & \\
\hline & KO DMSO & $62.6 \pm 6.8 \%$ & $10 / 3$ & $\begin{array}{c}\text { WT DMSO vs } \\
\text { KO NS }\end{array}$ & $>0.9999$ & \\
\hline & KO NS & $91.9 \pm 8.3 \%$ & $11 / 3$ & $\begin{array}{c}\text { WT DMSO vs } \\
\text { WT NS }\end{array}$ & $>0.9999$ & \\
\hline \multirow{2}{*}{ Fig4F } & DMSO & $100.0 \pm 4.1 \%$ & $13 / 3$ & \multirow{2}{*}{$\begin{array}{l}\text { DMSO vs } \\
\text { Paxilline }\end{array}$} & \multirow{2}{*}{0.6182} & \multirow{2}{*}{$\begin{array}{l}\text { Unpaired t- } \\
\text { test }\end{array}$} \\
\hline & Paxilline & $95.3 \pm 8.4 \%$ & $13 / 3$ & & & \\
\hline Figure S1 & Group & Mean \pm SEM & $\begin{array}{c}\mathrm{n}=\# \text { of } \\
\text { coverslips/ } \mathrm{N}=\# \\
\text { of neuronal } \\
\text { preparations }\end{array}$ & Comparison & $\mathrm{P}$ & Statistical test \\
\hline \multirow{2}{*}{ Figs1B } & WT & $46.9 \pm 2.8 \%$ & $7 / 3$ & \multirow{2}{*}{ WT vs KO } & \multirow{2}{*}{0.3123} & \multirow{2}{*}{$\begin{array}{l}\text { Unpaired t- } \\
\text { test }\end{array}$} \\
\hline & $\mathrm{KO}$ & $42.0 \pm 3.7 \%$ & $7 / 3$ & & & \\
\hline \multirow{2}{*}{ FigS1D } & WT & $42.7 \pm 2.8 \%$ & $8 / 2$ & \multirow{2}{*}{ WT vs KO } & \multirow{2}{*}{0.3809} & \multirow{2}{*}{$\begin{array}{l}\text { Unpaired t- } \\
\text { test }\end{array}$} \\
\hline & $\mathrm{KO}$ & $39.4 \pm 2.4 \%$ & $8 / 2$ & & & \\
\hline Figure S2 & Group & Mean \pm SEM & $\begin{array}{c}\mathrm{n}=\# \text { of } \\
\text { coverslips/ } \mathrm{N}=\# \\
\text { of neuronal } \\
\text { preparations }\end{array}$ & Comparison & $\mathrm{P}$ & Statistical test \\
\hline \multirow{2}{*}{ FigS2A } & WT & $2123.0 \pm 291.3$ puncta & $14 / 3$ & \multirow{2}{*}{ WT vs KO } & \multirow{2}{*}{0.5382} & \multirow{2}{*}{$\begin{array}{l}\text { Unpaired t- } \\
\text { test }\end{array}$} \\
\hline & $\mathrm{KO}$ & $2372.0 \pm 274.1$ puncta & $14 / 3$ & & & \\
\hline Figure S3 & Group & Mean \pm SEM & $\begin{array}{c}\mathrm{n}=\text { \# coverslips } / \\
\mathrm{N}=\text { \# of neuronal } \\
\text { preparation, }\end{array}$ & Comparison & $\mathrm{P}$ & Statistical test \\
\hline \multirow{2}{*}{ FigS3A } & WT & $37056 \pm 2322 \mathrm{~nm}$ & $\mathrm{n} 8 / 3$ & \multirow{2}{*}{ WT vs KO } & \multirow{2}{*}{0.6743} & \multirow{2}{*}{$\begin{array}{l}\text { Two-way } \\
\text { ANOVA }\end{array}$} \\
\hline & $\mathrm{KO}$ & $35468 \pm 2812 \mathrm{~nm}$ & $\mathrm{n} 9 / 3$ & & & \\
\hline
\end{tabular}

Table S1. Statistical information 